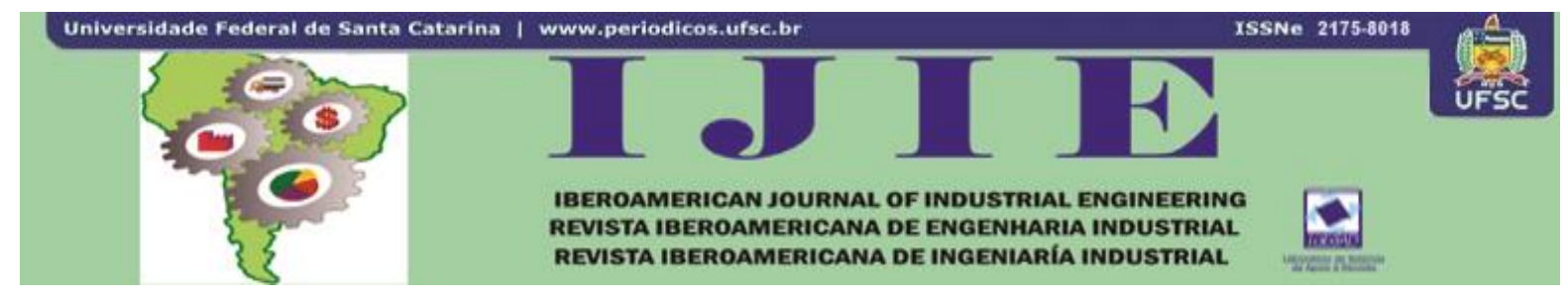

\title{
OPTIMIZACIÓN EN TIEMPO REAL CON DISYUNCIONES LÓGICAS: APLICACIÓN A SISTEMAS DE CALOR Y POTENCIA
}

\author{
Fernán Serralunga ${ }^{1}$ \\ Miguel Ceferino Mussati \\ Pio Antonio Aguirre ${ }^{3}$
}

RESUMEN: La Optimización en Tiempo Real (RTO) permite operar en cercanías del óptimo económico de una planta, respetando restricciones y respondiendo a cambios en el proceso. La adaptación con términos modificadores de las restricciones y los gradientes corrige eficientemente los errores estructurales de los modelos de RTO. Este trabajo propone el uso de RTO incluyendo disyunciones lógicas en el problema de optimización, y plantea una estrategia de adaptación con modificadores para este problema. La estrategia se muestra a través de un caso de estudio que consiste en un sistema de generación de vapor y potencia. Los resultados obtenidos muestran la validez de la estrategia sugerida y la utilidad de incluir variables discretas o disyunciones en los sistemas de RTO.

Palabras clave: Optimización en tiempo real. Adaptación por modificadores. Optimización de la energía.

\section{INTRODUCCIÓN}

La gestión eficiente del uso de la energía, reduciendo su consumo, respetando restricciones ambientales y minimizando los costos de operación, es un objetivo de vital importancia en los procesos industriales. El uso de técnicas asistidas por computadora en las etapas de síntesis, diseño y operación de los sistemas de calor y potencia resulta indispensable para alcanzar este objetivo.

\footnotetext{
${ }^{1}$ Ingeniero Químico, Instituto de Desarrollo y Diseño, Consejo Nacional de Investigaciones Científicas y Técnicas, Universidad Tecnológica Nacional (INGAR - CONICET - UTN). Avellaneda 3657 (S3002GJC) Santa Fe, Argentina. E-mail: fernanserralunga@gmail.com.

2 Dr., en Ingeniería Química, Instituto de Desarrollo y Diseño, Consejo Nacional de Investigaciones Científicas y Técnicas, Universidad Tecnológica Nacional (INGAR - CONICET - UTN). Avellaneda 3657 (S3002GJC) Santa Fe, Argentina. E-mail: mmussati@santafe-conicet.gov.ar.

${ }^{3}$ Dr., en Ingeniería Química. INGAR Instituto de Desarrollo y Diseño, Consejo Nacional de Investigaciones Científicas y Técnicas, Universidad Tecnológica Nacional (INGAR - CONICET - UTN). Avellaneda 3657 (S3002GJC) Santa Fe, Argentina. E-mail: paguir@santafe-conicet.gov.ar.
}

Iberoamerican Journal of Industrial Engineering, Florianópolis, SC, Brasil, v. 5, n. 10, p. 189202, 2013. 
Dentro de las técnicas utilizadas para optimizar la operación de una planta, la optimización en tiempo real (RTO) hace uso de un modelo y de las mediciones disponibles, y determina el valor que deben tomar las variables independientes de un proceso (entradas) para minimizar una función de costo. Los valores óptimos de las entradas son enviados como consigna al sistema de control, o como targets a un controlador predictivo multivariable (MPC). La frecuencia de ejecución de la optimización en tiempo real está en el orden de minutos u horas (DARBY et al., 2011).

Los errores del modelo (diferencias entre la predicción y el comportamiento real de la planta) pueden llevar a que la aplicación de las soluciones del RTO converja a un punto diferente del óptimo real de la planta, o incluso a que la solución no sea factible en la práctica. Estos errores pueden dividirse en paramétricos (los parámetros utilizados en el modelo no coinciden con los de la planta real) y estructurales (aún con los parámetros óptimos, las funciones utilizadas en el modelo no representan la operación de la planta) (CHACHUAT; SRINIVASAN; BONVIN, 2009).

Como estos dos tipos de errores están presentes en todos los modelos, las aplicaciones de RTO utilizan siempre alguna estrategia de adaptación del modelo para actualizarlo utilizando las mediciones disponibles. La estrategia tradicional (two-step approach) realiza dos cálculos de optimización (CHEN; JOSEPH, 1987): en el primero, actualiza los parámetros del modelo minimizando el error de las predicciones con respecto a los valores medidos; en el segundo, realiza la minimización de la función objetivo. En general, esta técnica soluciona el error paramétrico, y bajo ciertas condiciones, puede garantizar factibilidad (es decir, que la solución es factible en la práctica).

Otras técnicas incluyen la determinación experimental de los gradientes de la función objetivo y las restricciones, con el fin de corregir el error estructural y lograr que, cuando el sistema de RTO converge, el punto alcanzado sea un óptimo local de la planta (cumpliendo las condiciones de Karush-Kuhn-Tucker en la planta real). La técnica de ISOPE (Integrated System Optimization and Parameter Estimation) (ROBERTS, 1995) utiliza la técnica twostep approach pero agrega términos de corrección del gradiente a la función objetivo.

La técnica de adaptación por modificadores (modifier adaptation) (MARCHETTI, 2013) corrige las restricciones activas y la función objetivo, agregando además un bias a las restricciones; los parámetros del modelo permanecen constantes y se evita el primer paso de optimización.

Iberoamerican Journal of Industrial Engineering, Florianópolis, SC, Brasil, v. 5, n. 10, p. 189202, 2013. 
La determinación experimental de los gradientes puede realizarse perturbando las entradas del proceso y registrando cómo afectan a las variables de salida y las restricciones, o bien utilizando los resultados de los ciclos anteriores de RTO. Esta segunda opción requiere agregar restricciones al modelo para garantizar que el problema de estimación del gradiente esté bien condicionado, y recibe el nombre de dual modifier adaptation (MARCHETTI; CHACHUAT; BONVIN, 2010; MARCHETTI, 2013). Para una revisión de las técnicas de estimación de gradientes, pueden consultarse los trabajos de Mansour y Ellis (2003) y Srinivasan et al. (2011).

Los sistemas de servicios auxiliares presentan características para las cuales la aplicación de la optimización en tiempo real resulta conveniente. La variabilidad horaria de los precios de la electricidad, los cambios en la demanda de vapor en distintos niveles de presión, los cambios en la eficiencia de las turbinas de gas con la temperatura ambiente, y las restricciones ambientales y operativas, hacen que la operación óptima sea diferente en distintos horarios del día, y difícil de prever o planificar. Algunos autores han abordado el problema sin tener en cuenta la adaptación del modelo (VELASCO-GARCIA, 2011); otros han propuesto la optimización en tiempo real de una caldera aislada (YIP; MARLIN, 2004). Existen aplicaciones industriales para RTO y soporte de decisiones de sistemas de energía (MARIANI; KIHN; RUIZ, 2009).

El presente trabajo propone el uso de una técnica del tipo de adaptación por modificadores para la optimización en tiempo real de sistemas de energía. Todas las referencias mencionadas anteriormente suponen la resolución de problemas NLP; en esta contribución se plantea un problema que incluye disyunciones lógicas, y que puede plantearse como un problema de programación disyuntiva (GDP) o programación mixta entera no lineal (MINLP).

La aplicación de la propuesta se muestra en un caso de estudio en el que se simula la aplicación del sistema de RTO a un sistema de generación de vapor y potencia que incluye turbinas de gas con recuperación de calor y postcombustión, calderas convencionales, turbinas de vapor y otros consumos. El caso de estudio muestra la factibilidad de la propuesta, sus ventajas en comparación con las técnicas de RTO tradicional.

\section{OPTIMIZACIÓN EN TIEMPO REAL CON VARIABLES DISCRETAS}

\subsection{Inclusión de variables discretas en RTO}

Iberoamerican Journal of Industrial Engineering, Florianópolis, SC, Brasil, v. 5, n. 10, p. 189202, 2013. 
Se mencionó anteriormente que los problemas de RTO suelen ser del tipo NLP. Los motivos por los que no se incluyen variables enteras o lógicas se deben a que la RTO requiere que la solución se obtenga en tiempos cortos, mientras que un problema MINLP podría resolverse en varios minutos o horas; y que, en general, las soluciones a aplicar en el sistema de control son consignas de variables continuas, lo que no dejaría lugar a decisiones discretas. La puesta en marcha o parada de equipos, por seguridad operativa, no podría formar parte de la RTO y aplicarse automáticamente, sino que requiere la acción del personal de la planta.

Ahora bien, con la evolución de los algoritmos de optimización y de la velocidad de procesamiento de datos, el tiempo de ejecución puede dejar de ser un inconveniente. Con respecto a la utilidad de incluir disyunciones lógicas en el modelo, pueden mencionarse 3 potenciales usos:

1. Variables cuya consigna pueda modelarse como una disyunción. Ejemplo: una válvula cuya apertura puede estar entre $5 \%$ y $100 \%$, o bien totalmente cerrada.

2. Modelado de contratos complejos para la función costo. Ejemplo: penalidad en el consumo de electricidad o combustible, que incluye un costo fijo cuando el consumo supera un cierto umbral más un costo variable en función de la cantidad consumida por encima de dicho umbral.

3. Uso de diferentes modelos para diferentes rangos de operación de un equipo.

\subsection{Planteo del problema}

De acuerdo con lo sugerido en trabajos anteriores (SERRALUNGA; MUSSATI; AGUIRRE, 2012a, 2012b), se propone una estructura en la que se supone que los balances de materia, energía y entropía son conocidos. El error paramétrico y estructural está incluido en su totalidad en ecuaciones que predicen índices de eficiencia o desempeño de equipos, y que se suponen obtenidas empíricamente. Como ejemplo de estas ecuaciones empíricas, se puede mencionar la eficiencia de calderas y turbinas en función del caudal y el heat rate de una turbina de gas en función de la potencia generada. El problema a resolver puede expresarse de la siguiente manera (Ecuación 1):

Iberoamerican Journal of Industrial Engineering, Florianópolis, SC, Brasil, v. 5, n. 10, p. 189202, 2013. 


$$
\begin{aligned}
& \min _{\mathbf{u}} Q(\mathbf{y}, \mathbf{u})+\sum_{d} c_{d} \\
& \text { s.t. } \mathbf{h}(\mathbf{y}, \mathbf{u}, \boldsymbol{\eta})=0 \\
& \boldsymbol{\eta}_{G}=\mathbf{p}_{G}(\mathbf{u}) \\
& \mathbf{g}(\mathbf{y}, \mathbf{u}) \leq 0 \\
& {\left[\begin{array}{l}
z_{d} \\
\mathbf{h}_{d}(\mathbf{y}, \mathbf{u}, \boldsymbol{\eta})=0 \\
\eta_{d}=p_{d}(\mathbf{u}) \\
\mathbf{g}_{d}(\mathbf{y}, \mathbf{u}) \leq 0 \\
c_{d}=\gamma_{d}
\end{array}\right] \vee\left[\begin{array}{c}
\neg z_{d} \\
\left(\begin{array}{l}
\mathbf{y} \\
\mathbf{u} \\
\eta_{d}
\end{array}\right)=\mathbf{0} \\
c_{d}=0
\end{array}\right], d \in D} \\
& \boldsymbol{\Omega}(\mathbf{z}) \leq \mathbf{0} \\
& \mathbf{u}_{\mathbf{l}} \leq \mathbf{u} \leq \mathbf{u}_{u}, \mathbf{z} \in\{\text { verdadero, falso }\}^{m}
\end{aligned}
$$

donde $\mathbf{u}$ son las entradas al sistema, e $\mathbf{y}$ son las salidas. $z_{d}$ hace referencia a una disyunción, y $\mathbf{z}$ el vector que contiene todas las $z_{d}$. $\Omega$ es el conjunto de restricciones lógicas que relacionan las diferentes disyunciones. Q es la función objetivo, a la que se agregan los $\operatorname{costos}$ fijos $c_{d}$, que toman valor 0 cuando $z_{d}$ es falso, y $\gamma_{d}$ cuando $z_{d}$ es verdadero.

$\boldsymbol{\eta}=\left(\boldsymbol{\eta}_{G}, \eta_{d}, d \in D\right)^{\mathrm{T}}$ es el conjunto de índices de eficiencia incluidos en el modelo; $\boldsymbol{\eta}_{G}$ está presente en todos los casos, y $\eta_{d}$ sólo cuando $z_{d}=$ verdadero. Como no se conoce la funcionalidad real de estos índices con las entradas del modelo, se cuenta con un modelo aproximado $\boldsymbol{\eta}=\mathbf{p}(\mathbf{u})$.

$\mathbf{h}$ y $\mathbf{h}_{d}$ son los balances de materia, energía y entropía. Se consideran estructuralmente correctas, incluso cuando pueden incluir algún índice de eficiencia. Por ejemplo, el siguiente balance de energía en una caldera es estructuralmente correcto (Ecuación 2):

$$
h_{c}=Q . \eta_{c} / 100+F_{w} \cdot H_{w}-F_{v} \cdot H_{v}-F_{p} \cdot H_{p}=0
$$

donde $Q$ es el calor aportado por el combustible, $\eta_{c}$ es la eficiencia de la caldera, $\mathrm{F}$ y $\mathrm{H}$ son caudales y entalpías, respectivamente, y los subíndices $v, p$ y $w$ hacen referencia al vapor, la purga y el agua de calderas, respectivamente. Sin embargo, una ecuación de origen empírico para predecir la eficiencia en función del caudal como la siguiente (Ecuación 3):

$$
\eta_{c}=p_{c}\left(F_{v}\right)=a_{0}+a_{1} \cdot F_{v}
$$

con $a_{0}$ y $a_{l}$ constantes, incluye inevitablemente un error estructural al no predecir exactamente el comportamiento real de la caldera. 


\subsection{Adaptación del modelo}

Para adaptar el modelo utilizando las mediciones disponibles, sólo deben corregirse las ecuaciones que predicen los factores de eficiencia $\boldsymbol{\eta}$, ya que el resto de las ecuaciones se consideran libres de error.

El método propuesto para corregir el modelo se basa en la técnica de modifier adaptation, estimando los gradientes en base a los datos actuales y los de los ciclos anteriores de RTO. Una desventaja de este método es su difícil aplicación a sistemas con un gran número de entradas, ya que para la estimación del gradiente se requiere información de $n u+1$ ejecuciones de RTO (siendo $n u$ el número de entradas).

Como se ha sugerido en (SERRALUNGA; MUSSATI; AGUIRRE, 2012b), los términos de eficiencia de equipos generalmente pueden calcularse como una función de un número reducido de variables (y no del total de variables de entrada en el sistema que se desea optimizar) (Ecuación 4):

$$
\begin{gathered}
p_{G, i}(\mathbf{u})=p_{G, i}\left(\mathbf{x}_{G, i}(\mathbf{u})\right) \quad i=1 . . n g \\
p_{d}(\mathbf{u})=p_{d}\left(\mathbf{x}_{d}(\mathbf{u})\right) \quad, d \in D
\end{gathered}
$$

donde $i$ es cada una de las eficiencias que no están incluidas en una disyunción, $n g$ es el número de estas eficiencias, y $\mathbf{x}_{\mathrm{G}, \mathrm{i}} \mathrm{y} \mathbf{x}_{d}$ son las variables en función de las cuales se expresa la eficiencia.

En procesos como los sistemas de energía que se analizan en este trabajo, las eficiencias son función de variables de entrada relacionadas con cada uno de los equipos, por lo que la dimensión de los vectores $\mathbf{x}_{\mathrm{G}, \mathrm{i}} \mathrm{y} \mathbf{x}_{d}$ es menor que $n u$, la dimensión del vector de entradas al sistema. Esto permite que el gradiente de cada una de las restricciones se calcule experimentalmente con respecto a un grupo reducido de variables. Como consecuencia, se necesitan menos datos para estimar cada uno de los gradientes, ampliando la aplicación de la técnica a sistemas con un gran número de entradas.

Para una ejecución $k$ de optimización en tiempo real, los pasos para adaptar el modelo son los siguientes:

1. Obtención de los índices de eficiencia reales de la planta $\boldsymbol{\eta}^{\text {real,k}}$, utilizando las mediciones de la planta.

Para esto, es necesario resolver el siguiente sistema de Ecuación 5:

Iberoamerican Journal of Industrial Engineering, Florianópolis, SC, Brasil, v. 5, n. 10, p. 189202, 2013. 


$$
\begin{aligned}
& \quad \mathbf{h}\left(\mathbf{y}^{m, k}, \mathbf{u}^{m, k}, \boldsymbol{\eta}^{\text {real }, k}\right)=0 \\
& \left(\begin{array}{l}
\text { if } z_{d}^{k}, \\
\mathbf{h}_{d}\left(\mathbf{y}^{m, k}, \mathbf{u}^{m, k}, \boldsymbol{\eta}^{\text {real }, k}\right)=0 \\
\text { else } \\
\eta_{d}=0 \\
\text { endif }
\end{array}\right), d \in D
\end{aligned}
$$

donde $\mathbf{y}^{m, k}$ y $\mathbf{u}^{m, k}$ son las entradas y salidas medidas del modelo, y $z_{d}^{k}$ es el valor actual de la disyunción $d$.

Como ejemplo, puede obtenerse la eficiencia actual mediante la Ecuación 3 si se cuenta con mediciones de caudal de combustible, caudales de vapor, agua y purga, y presiones y temperaturas de las corrientes para calcular la entalpía.

En caso de que haya redundancia en las mediciones, el problema puede plantearse como una reconciliación de datos, calculando el valor más probable para las variables medidas.

2. Cálculo del modificador $\alpha$ de cada ecuación de eficiencia (bias), a partir de la eficiencia real y la calculada con el modelo aproximado (Ecuación 6):

$$
\begin{aligned}
& \alpha_{G, i}^{k}=\eta_{G, i}^{\text {real }, k}-p_{G, i}\left(\mathbf{x}_{G, i}^{k}\right) \quad, i=1 . . n g \\
& \left(\begin{array}{l}
\text { if } z_{d}^{k}, \\
\alpha_{d}^{k}=\eta_{d}^{r e a l, k}-p_{d, j}\left(\mathbf{x}_{d}^{k}\right)=0 \\
\text { else } \\
\quad \alpha_{d}^{k}=\alpha_{d}^{k-1} \\
\text { endif }
\end{array}\right), d \in D
\end{aligned}
$$

3. Cálculo del modificador del gradiente, a partir de los valores del modificador actual y los anteriores. De acuerdo con (SERRALUNGA; MUSSATI; AGUIRRE, 2012a), la propuesta es obtener el bias a utilizar y el modificador del gradiente a través de una regresión lineal (Ecuación 7):

$$
\begin{aligned}
& a_{G, i}^{k}, \mathbf{b}_{G, i}^{k}=\arg \min \sum_{j=k-N_{G, i}-1}^{k}\left(\alpha_{G, i}^{j}-a_{G, i}-\mathbf{b}_{G, i}^{T} \cdot \mathbf{x}_{G, i}^{j}\right)^{2}, i=1 . . n g \\
& a_{d}^{k}, \mathbf{b}_{d}^{k}=\operatorname{argmin} \sum_{j=k-N_{d}-1}^{k} z_{d}^{j}\left(\alpha_{d}^{j}-a_{d}-\mathbf{b}_{d}^{T} \cdot \mathbf{x}_{d}^{j}\right)^{2}, d \in D
\end{aligned}
$$


$N_{G, i}$ y $N_{d}$ son los números de datos a utilizar en cada regresión. Pueden ser diferentes para cada una de las eficiencias, dependiendo de la dimensión del correspondiente vector $\mathbf{x}$ y de las características de los datos a ajustar en cada caso.

El problema de optimización en tiempo real a resolver en el ciclo $k$ resulta (Ecuación 8):

$$
\begin{aligned}
& \min _{\mathbf{u}} Q(\mathbf{y}, \mathbf{u})+\sum_{d} c_{d} \\
& \text { s.t. } \mathbf{h}(\mathbf{y}, \mathbf{u}, \boldsymbol{\eta})=0 \\
& \eta_{G}=p_{G, i}\left(\mathbf{x}_{G, i}\right)+a_{G, i}^{k}+\left(\mathbf{b}_{G, i}^{k}\right)^{T} \cdot \mathbf{x}_{G, i}^{m} \quad i=1 . . n g \\
& \mathbf{g}(\mathbf{y}, \mathbf{u}) \leq 0 \\
& \left.\left[\begin{array}{c}
z_{d} \\
\eta_{d}=p_{d}(\mathbf{y}, \mathbf{u}, \boldsymbol{\eta})=0 \\
\mathbf{x}_{d}(\mathbf{y}, \mathbf{u}) \leq 0 \\
c_{d}=r_{d}^{k}+\left(\mathbf{b}_{d}^{k}\right)^{T} \cdot \mathbf{x}_{d}^{m}
\end{array}\right] \vee\left[\begin{array}{c}
\neg z_{d} \\
\mathbf{y} \\
\mathbf{u} \\
\eta_{d}
\end{array}\right)=\mathbf{0}\right], d \in D \\
& \mathbf{\Omega}(\mathbf{z}) \leq \mathbf{0} \\
& \mathbf{u}_{\mathbf{l}} \leq \mathbf{u} \leq \mathbf{u}_{u}, \mathbf{z} \in\{\text { verdadero, falso }\}^{m}
\end{aligned}
$$

\subsection{Estructura de RTO propuesta}

Un sistema de RTO completo requiere de cinco etapas (DARBY, 2011; MANSOUR; ELLIS, 2008): Detección de estado estacionario, validación de datos (que puede incluir reconciliación de datos y detección de errores gruesos), adaptación del modelo (con alguna de las estrategias mencionadas anteriormente), optimización de la función objetivo, y validación de los resultados para ser enviados al sistema de control.

La estrategia propuesta para adaptar el modelo, corrigiendo eficiencias por equipos, permite descomponer la etapa de adaptación en módulos, cada uno correspondiente a un equipo o una parte del sistema a optimizar. Cada uno de estos módulos es el encargado de actualizar el modelo correspondiente.

La actualización de los modelos no necesariamente se realiza con la misma frecuencia que la RTO. Cada uno de los módulos puede tener una frecuencia de actualización diferente, aprovechando la información disponible en los tiempos ociosos entre los ciclos de RTO. Además, se pueden utilizar estrategias diferentes para actualizar los términos modificadores de cada curva de eficiencia $p_{G, i} o p_{d}$. La Figura 1 muestra un esquema de la estructura propuesta.

Iberoamerican Journal of Industrial Engineering, Florianópolis, SC, Brasil, v. 5, n. 10, p. 189202, 2013. 
Figura 1 - Estructura para la optimización en tiempo real

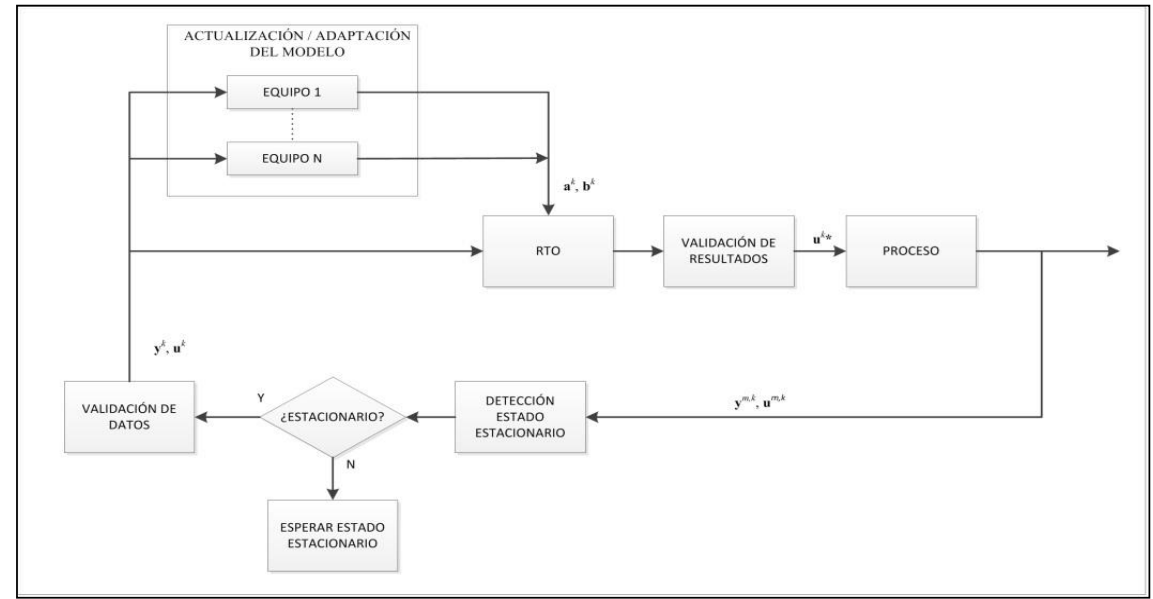

\section{CASO DE ESTUDIO}

\subsection{Descripción del caso}

El desempeño de la estructura de RTO propuesta se muestra a través de un sistema de generación de vapor y potencia, y una red que hace uso del vapor generado. El sistema cuenta con dos calderas, una turbina de gas que genera electricidad, una caldera de recuperación que aprovecha los humos de la turbina de gas, turbinas de vapor de contrapresión generando potencia mecánica, una turbina de condensación con extracción que genera electricidad, consumidores de vapor de baja presión y un desaireador para el tratamiento del agua de calderas. El diagrama del sistema de vapor y potencia modelado se muestra en la Figura 2. La función objetivo para la optimización en tiempo real es (Ecuación 9):

$$
\min C_{\text {comb }}\left(Q_{\text {cald } 1}+Q_{c a l d 2}+Q_{T G}+Q_{p c}\right)+C_{a g u a} F_{F W}+C_{E E}\left(D_{E}-W_{T G}-W_{\text {turbo }}\right)+\text { pen }
$$

donde $C_{\text {comb }}, C_{\text {agua }}$ y $C_{E E}$ son los costos de combustible, agua y electricidad, respectivamente, $Q$ son los consumos de combustible en calderas, turbina de gas y postcombustión, $F_{F W}$ es el consumo de agua, $D_{E}$ la demanda de electricidad y $W$ la producción eléctrica de la turbina de gas y el turbogenerador. Pen es la penalidad por consumo eléctrico mencionada anteriormente. El costo de combustible está fijo en 7.2 \$/GJ, y el de agua en 0.5 \$/t. 
Figura 2 - Esquema del sistema de vapor y potencia modelado

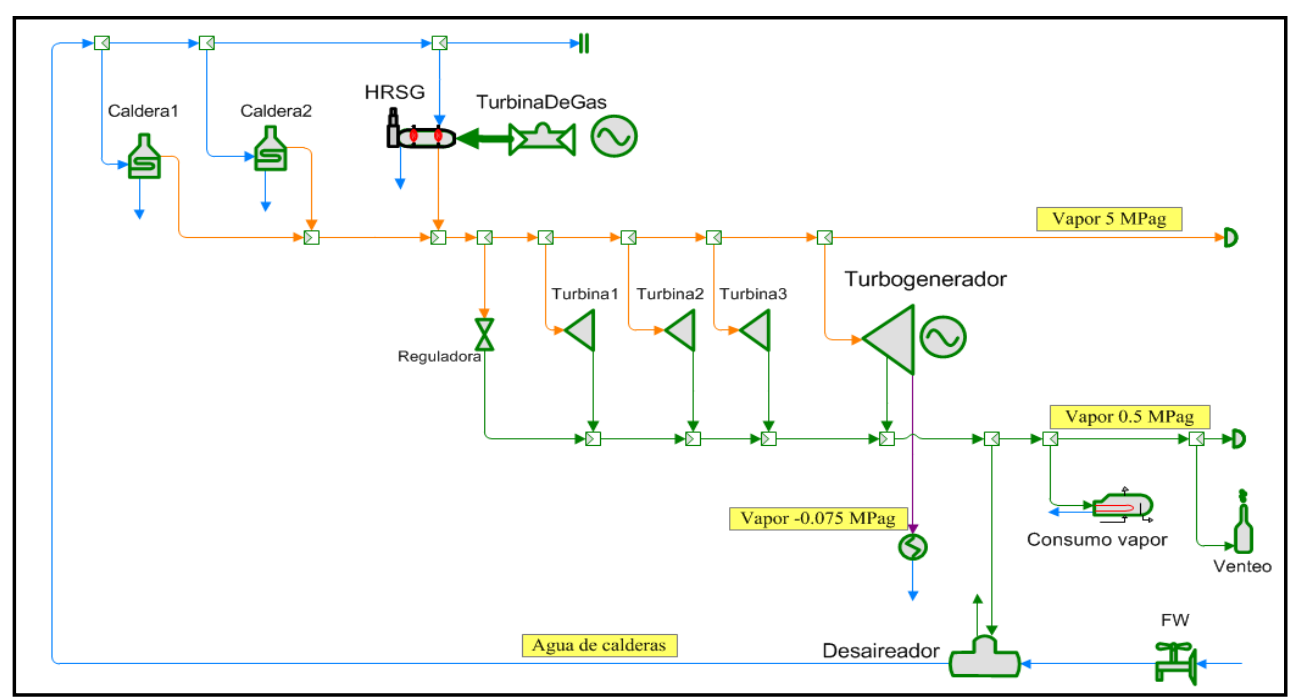

El caso a estudiar presenta dos características que pueden modelarse mediante disyunciones o variables discretas:

- Es posible generar vapor adicional en la caldera de recuperación quemando combustible en el conducto de humos provenientes de la turbina de gas. Esta producción extra de vapor es muy eficiente, ya que la turbina de gas trabaja con un alto exceso de aire, y el aire remanente queda disponible y a alta temperatura a la entrada de la caldera de recuperación. En este caso, se supone que es posible quemar gas natural en la postcombustión aportando entre 30 MW y 100 MW, o bien 0 MW.

- La compra de electricidad está penalizada cuando se superan los 11 MW consumidos. La penalidad incluye un término fijo que equivale al consumo de $1 \mathrm{MW}$ más un término variable por el consumo por encima de $11 \mathrm{MW}$. (Ejemplo: si se consumen 11.5 MW se paga penalidad por 1.5 MW). El precio de la penalidad es el doble del costo eléctrico variable $C_{E E}$.

Las variables continuas que se incluyen en el modelo son las producciones de vapor en las calderas, la generación de potencia en la turbina de gas y el caudal de admisión y el de extracción del turbogenerador.

La metodología utilizada para analizar el comportamiento de la RTO es la siguiente: Se cuenta con dos modelos del sistema de energía. Se asume que uno de ellos, llamado modelo real o planta, representa exactamente el comportamiento de la planta. El otro, llamado modelo aproximado, presenta diferencias estructurales con respecto al primer modelo. El

Iberoamerican Journal of Industrial Engineering, Florianópolis, SC, Brasil, v. 5, n. 10, p. 189202, 2013. 
modelo real se utiliza para simular el proceso, verificando la factibilidad de la solución propuesta por la RTO, calculando el costo real de operación, generando valores para la adaptación del modelo aproximado y calculando las condiciones óptimas de operación reales.

El modelo aproximado, corregido con el esquema de adaptación propuesto, se utiliza para la optimización en tiempo real, determinando los valores óptimos de las variables que se envían a la planta.

Las diferencias entre los dos modelos se resumen en la Tabla 1.

Tabla 1 - Diferencias entre el modelo aproximado y la planta. $F_{1}$ : Caudal vapor caldera $1 ; F_{2}$ : Caudal vapor caldera $2 ; F_{3}$ : Caudal vapor $1^{\circ}$ etapa turbogenerador; $F_{4}$ : Caudal vapor $2^{\circ}$ etapa turbogenerador;

$T$ : Temperatura ambiente

\begin{tabular}{lll}
\hline \multicolumn{1}{c}{ Índice de eficiencia } & \multicolumn{1}{c}{ Modelo aproximado } & \multicolumn{1}{c}{ Modelo real } \\
\hline Eficiencia Caldera 1 $(\%)$ & 92 & $75+0.51 F_{1}-0.0037\left(F_{1}\right)^{2}$ \\
Eficiencia Caldera 2 $(\%)$ & 92 & $75+0.51 F_{2}-0.0037\left(F_{2}\right)^{2}$ \\
Ef. ${ }^{\circ}$ etapa Turbogenerador $(\%)$ & $70-0.005\left(130-\mathrm{F}_{3}\right)^{2}$ & $70-140 /\left(1+F_{3}\right)$ \\
Ef. ${ }^{\circ}$ etapa Turbogenerador $(\%)$ & $70-0.004\left(150-\mathrm{F}_{4}\right)^{2}$ & $70-100 /\left(1+F_{4}\right)$ \\
Caudal/Potencia Turbina 1 $(\mathrm{kg} / \mathrm{kWh})$ & 12 & Eficiencia $=50 \%$ \\
Caudal/Potencia Turbina 2 $(\mathrm{kg} / \mathrm{kWh})$ & 12 & Eficiencia $=50 \%$ \\
Caudal/Potencia Turbina 3 $(\mathrm{kg} / \mathrm{kWh})$ & 12 & Eficiencia $=50 \%$ \\
Potencia máxima turbina de gas & $1.7 \mathrm{E}-6 T^{3}-2.72 \mathrm{E}-4 T^{2}-$ & $39+1.65 \mathrm{E}-6(T-15)^{5}-2 . \mathrm{E}-4(T-$ \\
(MW) & $0.24 T+43.68$ & $15)^{2}-2.43(T-15)$ \\
Heat Rate turbina de gas a carga & $1.5 .10^{-7} T^{3}+9.8 .10^{-5} T^{2}$ & $11+1.49 \mathrm{E}-7(T-15)^{5}+\quad 1 \mathrm{E}-$ \\
máxima $(\mathrm{GJ} / \mathrm{MWh})$ & $+0.012 T+10.59$ & $4(T-15)^{2}+0.016(T-15)$ \\
\hline
\end{tabular}

La adaptación del modelo se realiza siguiendo los pasos sugeridos en la Sección 2.3. Al cálculo de las eficiencias realizado en el paso 1, se agrega ruido gaussiano con una desviación estándar de $50 \mathrm{~kW}$ para la potencia máxima de la turbina de gas, $0.05 \mathrm{GJ} / \mathrm{MWh}$ para heat rate, $0.1 \%$ para la eficiencia en las calderas, $0.2 \%$ para las eficiencias en el turbogenerador y $0.3 \mathrm{t} / \mathrm{h}$ para el caudal de las turbinas. Las Ecuaciones adaptadas según la Ecuación (7) son las eficiencias de las calderas y el turbogenerador. En todos los casos el número de datos utilizado para la adaptación es 6. El resto de las Ecuaciones de la Tabla 1 se adaptan sin término corrector del gradiente.

Los modelos se han implementado en GAMS como modelos MINLP, y se resolvieron utilizando DICOPT.

\section{RESULTADOS Y DISCUSIÓN}

Se realizó una simulación de la evolución del sistema para 30 ejecuciones de RTO consecutivas, suponiendo costos, demanda de vapor y demanda de electricidad constantes, y

Iberoamerican Journal of Industrial Engineering, Florianópolis, SC, Brasil, v. 5, n. 10, p. 189202, 2013. 
sin ruido en las mediciones, para verificar la convergencia del método. La estrategia propuesta, y su comparación con la evolución utilizando la estrategia clásica de dos pasos.

Se observa que la estrategia propuesta converge al entorno del óptimo real de la planta, mientras que la estrategia de dos pasos converge a un costo operativo mayor. En ninguno de los casos se paga penalidad eléctrica. La estrategia de dos pasos trabaja con postcombustión en todos los ciclos, mientras que la propuesta evoluciona a no utilizar la postcombustión, en coincidencia con el óptimo real.

Figura 1 - Evolución para mediciones sin ruido. (a) Costo operativo . (b) Postcombustión. TS = Estrategia de dos pasos. $C_{E E} .=90 \$ / \mathrm{MWh}$. Temp. ambiente $=15^{\circ} \mathrm{C}$. Demanda vapor $=75 \mathrm{t} / \mathrm{h}$. Demanda elect. $=63 \mathrm{MW}$

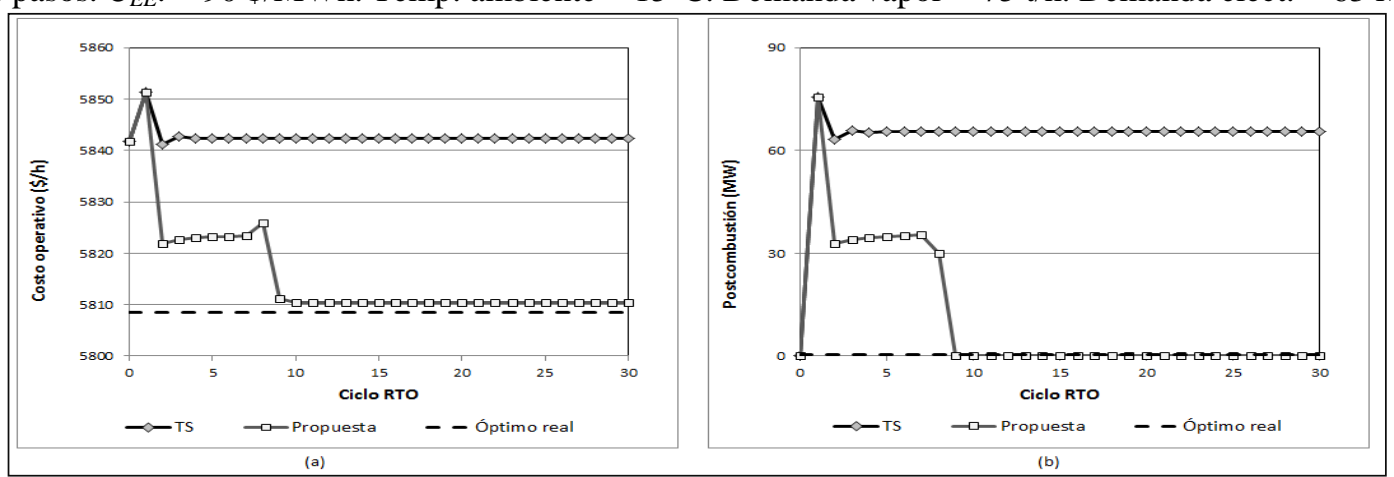

La Figura 4 muestra la evolución para 30 ejecuciones con cambios en la demanda de electricidad y vapor, precio de la electricidad y temperatura ambiente, incluyendo además el ruido gaussiano mencionado en la Sección 3.1. Puede observarse que la propuesta alcanza un costo operativo menor o igual a la estrategia de dos pasos, en todos los ciclos excepto en uno En este punto, el ruido en las mediciones y un cambio en la demanda de vapor causaron que la adaptación propuesta lleve al sistema a una condición más alejada del óptimo que la estrategia tradicional.

Figura 2(a) - Evolución del sistema a cambios en precios, demandas y temperatura ambiente. (b) Perfiles de temperatura, precio y demandas. (c) Postcombustión. TS= Estrategia de dos pasos

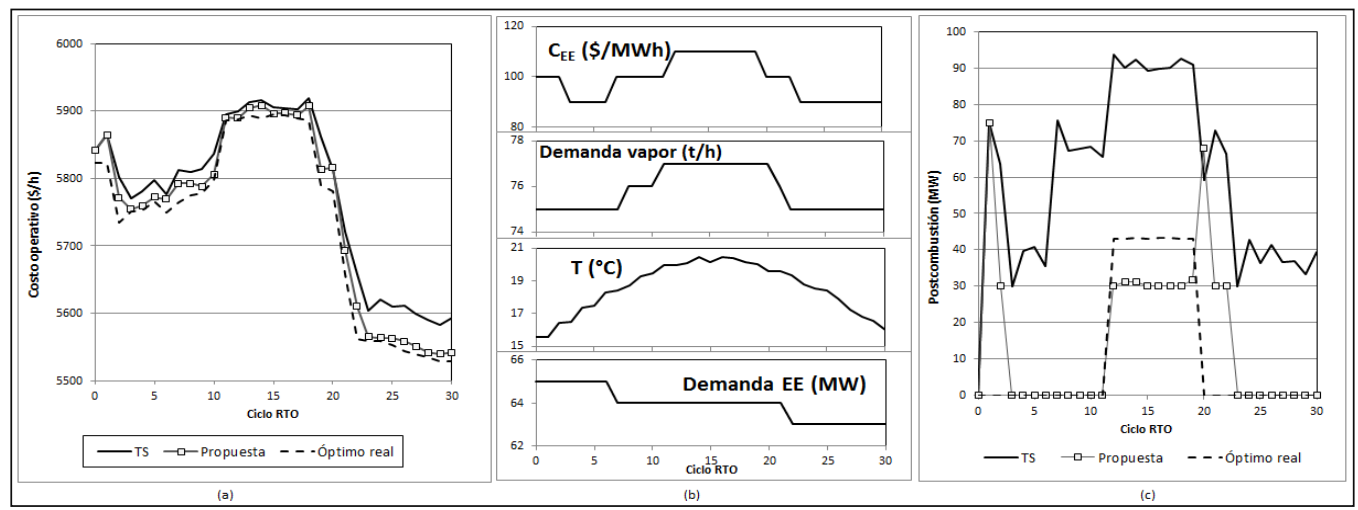

Iberoamerican Journal of Industrial Engineering, Florianópolis, SC, Brasil, v. 5, n. 10, p. 189202, 2013. 
Se observa también que la presencia de ruido y la variabilidad de las condiciones en el sistema afectan la performance de la metodología propuesta. Se observa que la estrategia de dos pasos utiliza la postcombustión en todos los casos, mientras que la propuesta coincide con el óptimo real en la decisión de utilizar o no postcombustión en 25 de los 30 casos. En todos los casos, ningún ciclo opera con penalizaciones.

Para comparar el desempeño del sistema de RTO propuesto con el tradicional, se calcula el error con respecto al óptimo real a lo largo de los ciclos, siguiendo la técnica Extended Design Cost (EDC) propuesta por Zhang y Forbes (2000). La Tabla 2 resume los errores en los dos casos estudiados.

Tabla 2 - Extended Design Cost para los casos estudiados. (TS = Estrategia de dos pasos) Caso 1: demanda y costos constantes, sin ruido. Caso 2: demanda y costos variables, con ruido

\begin{tabular}{ccc}
\hline Caso & EDC $(\mathbf{\$})$ & \% reducción EDC \\
\hline Caso 1 - TS & 1027 & \\
Caso 1 - Propuesta & 208 & 79.7 \\
Caso 2 - TS & 1236 & \\
Caso 2 - Propuesta & 471 & 61.9 \\
\hline
\end{tabular}

\section{CONCLUSIONES}

Este trabajo propone un esquema de adaptación de modelos para optimización en tiempo real en problemas que incluyen disyunciones o variables discretas. El esquema incluye la corrección de los gradientes para ecuaciones que calculan índices de eficiencia de equipos.

Este esquema se aplicó a un caso de estudio consistente en un sistema de vapor y potencia. Los resultados obtenidos muestran las ventajas comparativas de incluir la estrategia de adaptación propuesta con respecto a los métodos tradicionalmente utilizados en la industria.

Se observa que la presencia de ruido en las mediciones (inevitable en las aplicaciones reales) reduce la efectividad del método, al igual que los cambios en las condiciones del proceso en el lapso entre dos ejecuciones de RTO. La forma metodológica para abordar y resolver estos inconvenientes es un aspecto a tratar en un próximo trabajo.

\section{REAL-TIME OPTIMIZATION WITH LOGICAL DISJUNCTIONS: APPLICATION TO HEAT AND POWER SYSTEMS}


ABSTRACT: Real-time optimization (RTO) allows operating an industrial plant close to its (economic) optimal point, while respecting constraints and reacting to process disturbances. The structural plant-model mismatch can be efficiently corrected by the modifier adaptation strategy, which corrects errors in constraint values, as well as in cost and constraint gradients. This work proposes the inclusion of logical disjunctions in RTO, and suggests a modifier adaptation strategy for this structure. The strategy is shown through a case study which analyzes a heat and power system. The results obtained show the usefulness of including discrete variables or disjunctions in RTO systems, and prove the efficacy of the suggested adaptation strategy.

Keyword: Real-time optimization . Modifier adaptation. Energy optimization.

Agradecimientos: Los autores agradecen a CONICET y Soteica Latinoamérica S.A. por el apoyo financiero para realizar este trabajo.

\section{REFERENCIAS}

CHACHUAT, B., SRINIVASAN, B., BONVIN, D. Adaptation strategies for real-time optimization. Computers \& Chemical Engineering, v. 33, n. 10, p. 1557-1567, 2009.

CHEN, C.Y.; JOSEPH, B. On-line optimization using a two-phase approach: an application study. Industrial \& Engineering Chemistry Research, v. 26, n. 9, p. 1924-1930, 1987.

DARBY, M.L.; NIKOLAOU, M.; JONES, J.; NICHOLSON, D. RTO: an overview and assessment of current practice. Journal of Process Control, v. 21, n. 6, p. 874-884, 2011.

MANSOUR, M.; ELLIS, J.E. Comparison of methods for estimating real process derivatives in on-line optimization. Applied Mathematical Modelling, v. 27, n. 4, p. 275-291, 2003.

MANSOUR, M.; ELLIS, J.E. Methodology of on-line optimisation applied to a chemical reactor. Applied Mathematical Modelling, v. 32, n. 2, p. 170-184, 2008.

MARCHETTI, A.; CHACHUAT, B.; BONVIN, D. A dual modifier-adaptation approach for real-time optimization. Journal of Process Control, v. 20, n. 9, p. 1027-1037, 2010.

MARCHETTI, A.G. A new dual modifier-adaptation approach for iterative process optimization with inaccurate models. Computers \& Chemical Engineering, v. 59, p. 89100, 2013.

MARIANI, D.C.; KIHN, M.A.; RUIZ, C.A. Industrial Experience on the Implementation of Real Time On Line Energy Management Systems in Sugar and Alcohol Industry. Computer Aided Chemical Engineering, (10th International Symposium on Process Systems Engineering: Part A), v. 27, p. 459-464, 2009.

Iberoamerican Journal of Industrial Engineering, Florianópolis, SC, Brasil, v. 5, n. 10, p. 189202, 2013. 
ROBERTS, P.D. Coping with model-reality differences in industrial process optimization: a review of integrated system optimisation and parameter estimation. Computers in Industry, v. 26, p. 281-290, 1995.

SERRALUNGA, F.; MUSSATI, M.C.; AGUIRRE, P.A. An alternative real-time optimization algorithm with modifier adaptation: Application to heat and power systems. Computer Aided Chemical Engineering (22nd European Symposium on Computer Aided Process Engineering), v30, p. 367-371, 2012b.

SERRALUNGA, F.; MUSSATI, M.C.; AGUIRRE, P.A. Real-time optimization of energy systems in sugar and ethanol facilities: a modifier adaptation approach. Computer Aided Chemical Engineering (11th International Symposium on Process Systems Engineering), v31, p. 375-379, 2012a.

SRINIVASAN, B.; FRANÇOIS, G.; BONVIN, D. Comparison of Gradient Estimation Methods for Real-time Optimization. Computer Aided Chemical Engineering (21st European Symposium on Computer Aided Process Engineering), v29, p. 607-611, 2011.

VELASCO-GARCIA, P.; VARBANOV, P.S.; ARELLANO-GARCIA, H.; WOZNY, G. Utility systems operation: optimisation-based decision making. Applied Thermal Engineering, v. 31, p. 3196-3205, 2011.

YIP, W.S.; MARLIN, T.E. The effect of model fidelity on real-time optimization performance. Computers \& Chemical Engineering, v. 28, p. 267-280, 2004.

ZHANG, Y.; FORBES, J.F. Extended design cost: a performance criterion for real-time optimization systems. Computers \& Chemical Engineering, v. 24, p. 1829-1841, 2000.

Originais recebidos em: 26/10/2013

Aceito para publicação em: 15/04/2014

Iberoamerican Journal of Industrial Engineering, Florianópolis, SC, Brasil, v. 5, n. 10, p. 189202, 2013. 